\title{
Parasitoses Intestinales Et Statut Nutritionnel Chez L'enfant À Guédiawaye Au Sénégal
}

\section{Diouf Jean Baptiste Niokhor, Kane Mariama,}

Service de Pédiatrie de L'hôpital Roi Baudouin de Guédiawaye, Sénégal

\section{Sow Doudou,}

Service de Parasitologies - Section Biologie et Explorations Fonctionnelles UFR Sciences de la Santé, Université Gaston Berger, Saint Louis, Sénégal

Sougou Ndèye Marème,

Département de Santé Publique et Médecine Préventive

Université Cheikh Anta Diop de Dakar, Sénégal

Oumy Kaltome Boh, Babacar Faye,

Laboratoire Parasitologie, Université Cheikh Anta Diop de Dakar, Sénégal Ndiaye Ousmane,

Service de pédiatrie Université Cheikh Anta Diop de Dakar, Sénégal

Doi:10.19044/esj.2020.v16n15p115 URL:http://dx.doi.org/10.19044/esj.2020.v16n15p115

\section{Résumé}

Introduction : Les parasitoses intestinales constituent un problème majeur de santé dans le monde particulièrement dans les pays en voie de développement. Afin de réduire l'ampleur de ces affections, le Ministère de la Santé du Sénégal a introduit en 2005 le déparasitage de masse systématique des enfants conformément aux recommandations de l'Organisation Mondiale pour la Santé (OMS). L'objectif de ce travail était de déterminer la prévalence des parasitoses intestinales et leur impact sur l'anémie et la nutrition quelques années après l'instauration de la chimiothérapie préventive. Matériels et méthodes : Etude transversale du $1^{\mathrm{er}}$ septembre 2017 au 28 février 2018 au niveau du service de pédiatrie du Centre Hospitalier Roi Baudouin de Guédiawaye. Un effectif de 375 enfants avait participé à l'étude, avec un âge moyen 44 mois et une prédominance masculine $(54,1 \%)$. Pour chaque enfant reçu, un examen direct de selles et après une concentration par technique de Ritchie étaient effectués. L'état nutritionnel a été appréciés et les paramètres hématologiques évalués. Résultats : Au total, 103 enfants étaient porteurs de parasites soit une prévalence globale de 27,5\%. Les helminthes étaient beaucoup plus représentatifs que les protozoaires et les espèces parasitaires les 
plus fréquentes étaient Ascaris lumbricoides (18,14\%), Trichocéphales (3,74\%), et Entamoeba coli (2,67\%). L'état nutritionnel était normal chez 265 enfants tandis que $18,9 \%$ et $10,5 \%$ avaient respectivement une malnutrition aigüe modérée (MAM) et une malnutrition aigüe sévère (MAS). Nous avons noté une association significative entre la parasitose intestinale et la malnutrition $(\mathrm{p}=0,035$ et $\mathrm{OR}=1,66)$. L'anémie était sévère chez $4,8 \%$ des enfants et modérée chez $68,6 \%$. Une corrélation entre les parasitoses intestinales et la survenue d'une anémie a été démontrée ( $\mathrm{p}=0,001$ et $\mathrm{OR}=2,6$ ). Conclusion: La prévalence des parasitoses intestinales reste élevée en banlieue dakaroise malgré le déparasitage de masse. L'amélioration des conditions de vie et l'élargissement du déparasitage aux enfants de plus de 5 ans pourraient rendre plus efficiente cette stratégie.

Mots-clés : Parasitoses Intestinales, Anémie, Malnutrition, Enfants, Guédiawaye, Sénégal

\section{Intestinal Parasites and Nutritional Status in Children in Guédiawaye in Senegal}

\section{Diouf Jean Baptiste Niokhor, Kane Mariama,}

Service de Pédiatrie de L'hôpital Roi Baudouin de Guédiawaye, Sénégal

Sow Doudou,

Service de Parasitologies - Section Biologie et Explorations Fonctionnelles UFR Sciences de la Santé, Université Gaston Berger, Saint Louis, Sénégal

\section{Sougou Ndèye Marème,}

Département de Santé Publique et Médecine Préventive

Université Cheikh Anta Diop de Dakar, Sénégal

Oumy Kaltome Boh,

Babacar Faye,

Laboratoire Parasitologie, Université Cheikh Anta Diop de Dakar, Sénégal

Ndiaye Ousmane,

Service de pédiatrie Université Cheikh Anta Diop de Dakar, Sénégal

Abstract

Introduction: Intestinal parasitosis is a major health problem in the world, particularly in developing countries. In order to reduce the scale of 
these diseases, the Ministry of Health of Senegal introduced, in 2005, the systematic mass deworming of children in accordance with the recommendations of the World Health Organisation (WHO). This paper focuses on determining the prevalence of intestinal parasites and their impact on anemia and nutrition a few years after the initiation of preventive chemotherapy. Materials and methods: A cross-sectional study was conducted from 1st September 2017 to 28th February 2018 at the pediatric ward of the hospital center Roi Baudouin in Guédiawaye, Senegal. A total of 375 children participated in the study, with a mean age of 44 months and a male predominance $(54.1 \%)$. Each child received a direct stool examination after a concentration using Ritchie techniques was obtained. The nutritional status was assessed and the hematological parameters evaluated. Results: A total of 103 children had parasites, with an overall prevalence of $27.5 \%$. Helminths were much more representative than the protozoa, and the most common parasitic species were Ascaris lumbricoides (18.14\%), whipworms (3.74\%), and Entamoeba coli (2.67\%). Nutritional status was normal in 265 children, while $18.9 \%$ and $10.5 \%$ respectively had moderate acute malnutrition (MAM) and severe acute malnutrition (SAM). A significant association between intestinal parasitosis and malnutrition was found $(\mathrm{p}=$ 0.035 and $\mathrm{OR}=1.66$ ). Anemia was severe in $4.8 \%$ of children and moderate in $68.6 \%$. There was a link between intestinal parasitosis and the occurrence of anemia ( $p=0.001$ and $\mathrm{OR}=2.6)$. Conclusion: The prevalence of intestinal parasitosis remains high in the suburbs of Dakar despite mass deworming. Improving living conditions and expanding deworming to children over 5 years of age could make this strategy more efficient.

Keywords: Intestinal Parasites, Anemia, Malnutrition, Children, Guédiawaye, Senegal

\section{Introduction}

Les parasitoses intestinales constituent un problème majeur de santé dans le monde particulièrement dans les pays en voie de développement du fait de leur fréquence et de leur évolution continuelle. L'Organisation Mondiale de la Santé (OMS) estime à plus de 2 milliards le nombre de personnes atteintes dans le monde, avec un nombre annuel de décès estimé à 155000 cas par an (OMS, 2018). Chez les enfants d'âge scolaire des pays en développement, les parasitoses intestinales se situent au premier rang de toutes les maladies transmissibles et non transmissibles, compte tenu de la charge morbide qu'elles représentent (Mentresor, 2004). Au Sénégal, les résultats de l'enquête de base effectuée en 2010 par l'OMS révèlent que le taux global de prévalence des infections parasitaires intestinales est de 21,9\%. Les parasites intestinaux sont groupés en protozoaires et en helminthes. Leurs localisations 
dans l'organisme déterminent les diverses pathologies qu'elles peuvent causer. Les infections par les helminthes et les protozoaires représentent 25\% de l'ensemble des maladies infectieuses dans le monde (Mentresor, 2004). Les conséquences de ces affections sur la santé des enfants sont variables et peuvent être graves : anémie, retard de croissance, malnutrition, baisse du rendement scolaire (Alum, 2010). L'ampleur des parasitoses intestinales ainsi que leurs conséquences néfastes sur la survie de l'enfance, justifient la nécessité de mettre en œuvre des stratégies visant à réduire la morbidité liée à ces affections. C'est ainsi que l'OMS en 2001 a recommandé la promotion du déparasitage de masse systématique des enfants de moins de 5 ans comme stratégie de prévention et de réduction de leur impact négatif sur la santé des enfants (OMS, 2018). Conformément à cette directive, le Sénégal a mené des campagnes de déparasitage dans des districts cibles à partir de 2003 avec passage à l'échelle à partir de 2005 au profit des enfants de 6 à 59 mois (Ministère de la santé et de l'action sociale du Sénégal, 2011). C'est dans ce contexte que s'inscrit cette étude dont l'objectif principal est de décrire la prévalence des parasitoses intestinales et leur impact sur l'anémie et la malnutrition chez les enfants de moins de 15 ans reçus en consultation au service de pédiatrie de l'hôpital Roi Baudouin situé dans le district de Guédiawaye.

\section{Matériels et méthodes}

Cadre d'étude

L'étude était réalisée au sein du service de pédiatrie du Centre Hospitalier Roi Baudouin. Cette structure sanitaire est localisée à Guédiawaye qui constitue l'un des quatre départements de la région de Dakar. Il est situé à $17 \mathrm{~km}$ du centre-ville de la capitale dans une zone périurbaine localisée dans les Niayes avec un climat de type sub-sahélien. Ce structure dispose également d'un laboratoire où sont réalisées les analyses hématologiques, parasitologiques et bactériologiques.

\section{Schéma et population d'étude}

Une enquête transversale était réalisée sur une période de 6 mois allant du $1^{\text {er }}$ septembre 2017 au 28 février 2018. Un échantillonnage avec un pas de sondage était effectué chez les enfants reçus en consultation au service de pédiatrie.

\section{Taille d'échantillon et plan de sondage}

Si on se base sur une prévalence des helminthes dans la zone estimée à $20 \%$ et la formule de calcul de la taille d'échantillon fournie par l'OMS (=50/ ( 0.80 x prévalence en \%), la taille prévue de l'échantillon est 375 enfants. 


\section{Recueil des données}

Les enquêtes étaient effectuées à l'aide de questionnaires électroniques renseignant sur les aspects sociodémographiques des patients, les aspects cliniques, le statut nutritionnel et les résultats issus des examens biologiques. Les données recueillies sont saisies sur Excel.

\section{Examens parasitologiques des selles (Adam, 1971)}

Les selles étaient recueillies dans des pots appropriés propres hermétiquement fermés, étiquetés et aussitôt acheminés au laboratoire.

Tous les prélèvements de selles étaient examinés par deux microscopistes différents en procédant aux étapes suivantes :

Examen macroscopique : Il précise l'aspect de la selle (molle, dure, diarrhéique...), la présence ou non de parasites visibles à l'œil nu mais aussi la présence ou l'absence de mucus, de sang.

Examen microscopique direct en eau physiologique : Il permet de mettre en évidence des formes végétatives ou kystiques de protozoaires ou des œufs et larves d'helminthes. Il est fait à partir d'un peu de selles étalées dans une goutte d'eau salée à $9 \%$. La totalité de la préparation doit être examinée au grossissement 10 , puis au 40 .

Examen microscopique après les techniques de MIF (Merthiolate Iode Formol), de Kato katz et de la coloration de Zielh Neelsen modifiée.

La technique MIF permet de colorer les parasites en deux phases. Tout d'abord l'iode colore immédiatement les trophozoïtes qui apparaissent alors vert-jaune à jaune-brun. Au bout de quelques heures, les membranes nucléaires sont colorées en rouge foncé et le cytoplasme en rouge (Adam, 1971).

La méthode de Ziehl-Neelsen modifiée par Henriksen et Pohlenz issue de la coloration de Gram. Elle comporte une étape de fixation, puis une étape de coloration, une étape de décoloration et une dernière étape de recoloration. Elle permet l'identification des coccidies intestinales (Henriksen \& Pohlenz, 1981).

La méthode de Ritchie ou Ritchie simplifiée permet de mettre en évidence les œufs d'helminthes et les kystes de protozoaires mais le culot est souvent très abondant. Cette technique peut être suivie d'une coloration par le lugol pour faciliter la lecture des parasites (Mc Nabb, 1985).

\section{Mesure du taux d'hémoglobine :}

Le prélèvement était réalisé de la manière suivante : (a) du sang capillaire était prélevé au moyen d'une piqûre au doigt faite à l'aide d'une petite lance rétractable (Tenderlette) ; (b) une ou deux gouttes de sang était recueillie sur une cuvette miniature puis placée ensuite dans un 
hémoglobinomètre portatif (HemoCue), appareil qui, en moins d'une minute, pouvait donner une mesure exacte du taux (en grammes) d'hémoglobine par décilitre de sang.

Paramètres étudiés

Les variables suivantes étaient étudiées :

- Age en mois qui été défini en quatre catégories : moins de 12 mois; entre 12 et 60 mois ; entre 60 et 120 mois et entre 120 et 180 mois.

- Le sexe

- Le poids

- La taille

- L'état nutritionnel est apprécié selon les critères de l'OMS :

$>$ Malnutrition aigüe modérée (MAM) :

- Poids pour Taille entre -3 et - 2 Z-scores

Malnutrition aiguë sévère (MAS) :

- Indice Poids pour Taille <-3 Z- scores

- Et/ou la présence d'œdèmes bilatéraux

- Le taux d'hémoglobine

L'anémie simple et l'anémie sévère sont définies selon les critères de l'OMS par des taux d'hémoglobine inférieurs à $12,0 \mathrm{~g} / \mathrm{dl}$ et $8,0 \mathrm{~g} / \mathrm{dl}$ respectivement.

- Les résultats de l'examen parasitologique des selles.

\section{Analyses statistiques}

Les données sont analysées avec le logiciel R2.15.0 (R Foundation for statistical computing, Vienna, Austria). Pour les enquêtes de prévalence, les variables qualitatives sont été décrites en termes d'effectif, de pourcentage de données renseignées et les intervalles de confiance à $95 \%$ calculés. Les variables quantitatives ont été décrites en termes de moyenne. Les comparaisons statistiques faites en utilisant le test de $\mathrm{Khi}^{2}$ ou le test de Fisher en fonction des conditions d'applicabilité. Le test est considéré comme significatif si $p$ est inférieur à 0,05 .

\section{Aspects éthiques et réglementaires}

La présente étude faisait partie d'un projet de recherche financé par le centre d'excellence pour la santé de la mère et de l'enfant. Le protocole d'étude avait reçu un avis favorable du comité éthique sous le numéro Protocole 0241/2017/CER/UCAD. Un consentement est recueilli auprès du parent individuellement dans le box de consultation.

Aucun enfant dont les parents avaient opposé un refus à participer aux investigations prévues dans le cadre de l'étude, n'a été inclus. Les informations individuelles concernant les participants à l'étude, ont été 
analysées dans la confidentialité sans tenir compte de l'identité des participants.

\section{Résultats}

\section{Caractéristiques de la population d'étude}

Au total, 375 enfants âgés de moins de 15 ans avaient participé à l'étude. L'âge moyen des participants était de 44 mois. Il y avait une prédominance masculine $(54,1 \%)$ avec un sex ratio de 1,14 . Les symptômes gastrointestinaux étaient retrouvés dans $60,8 \%$ des cas et la fièvre dans $70,1 \%$ des cas (Tableau 1).

Tableau 1. Répartition de la population de l'étude selon les signes cliniques

\begin{tabular}{lll}
\hline Signes cliniques & Effectif & $\begin{array}{l}\text { Pourcenta } \\
\text { ge }\end{array}$ \\
\hline Signes gastro intestinaux & & \\
$\quad$ Douleurs abdominales & 86 & 22,9 \\
Diarrhée & 45 & 12,0 \\
$\quad$ Vomissement & 41 & 10,9 \\
Anorexie & 40 & 10,7 \\
Constipation & 16 & 4,3 \\
Autres signes cliniques & & \\
Fièvre & 263 & 70,1 \\
Respiratoires & 206 & 54,9 \\
Cutanées & 18 & 4,8 \\
Neurologiques & 9 & 2,4 \\
Cardiaques & 1 & 0,3 \\
$\quad$ Urinaires & 1 & 0,3 \\
\hline
\end{tabular}

Prévalence des parasitoses intestinales, de la malnutrition et de l'anémie Sur les 375 enfants reçus en consultation, 103 étaient parasités, soit une prévalence $27,5 \%$. Les espèces parasitaires les plus représentées étaient Ascaris lumbricoides, Trichocéphales, et Entamoeba coli.

Tableau 2. Fréquence globale du portage de parasites intestinaux

\begin{tabular}{lll}
\hline Paramètres & Effectif / N & Pourcentage \\
\hline $\begin{array}{l}\text { Enfants porteurs de parasites } \\
\text { intestinaux }\end{array}$ & $103 / 375$ & 27,5 \\
$\begin{array}{l}\text { Espèces parasitaires identifiées } \\
\text { Ascaris lumbricoides }\end{array}$ & $68 / 375$ & 18,14 \\
Trichocéphales & $14 / 375$ & 3,74 \\
Entameaba coli & $10 / 375$ & 2,67 \\
Ascaris lumbricoides & & \\
Trichocéphales & & \\
Blastocystis hominis & $09 / 375$ & 2,40 \\
& $02 / 375$ & 0,55 \\
\hline
\end{tabular}


Concernant l'état nutritionnel, $70,7 \%$ des enfants avaient un état nutritionnel normal, $18,9 \%$ une malnutrition aigüe modérée et $10,5 \%$ une malnutrition aigüe sévère.

Une anémie était identifiée chez 275 enfants dont $257(68,6 \%)$ présentaient une anémie modérée et $18(4,8 \%)$ une anémie sévère.

Tableau 3. Prévalence des parasitoses intestinales, de l'anémie et de la malnutrition

\begin{tabular}{|c|c|c|}
\hline Variables & Effectif & Pourcentage \\
\hline Parasitose intestinale & 103 & 27,5 \\
\hline $\begin{array}{l}\text { Ascaris lumbricoides } \\
\text { Trichocéphales } \\
\text { Entameaba coli } \\
\text { Ascaris lumbricoides } \\
\text { Trichocéphales } \\
\text { Blastocystis hominis }\end{array}$ & $\begin{array}{r}68 \\
14 \\
10 \\
+\quad 09 \\
\\
\end{array}$ & $\begin{array}{l}18,14 \\
3,74 \\
2,67 \\
2,40 \\
0,55\end{array}$ \\
\hline \multicolumn{3}{|l|}{ Anémie } \\
\hline Non anémiés & 100 & 26,5 \\
\hline Anémie modérée & 257 & 68,6 \\
\hline Anémie sévère & 18 & 4,8 \\
\hline \multicolumn{3}{|l|}{ Etat nutritionnel } \\
\hline Normal & 265 & 70,7 \\
\hline MAM & 71 & 18,9 \\
\hline MAS & 39 & 10,5 \\
\hline
\end{tabular}

\section{Facteurs associés aux parasitoses intestinales}

Parmi les enfants parasités, 28,7\% étaient âgés de 1 à 5 ans et il a été noté un lien significatif entre la survenue d'une parasitose intestinale et les différentes tranches d'âge ( $\mathrm{p}=0,007)$.

De même, il été noté une association significative entre la parasitose intestinale et la malnutrition ( $\mathrm{p}=0,035$ et $\mathrm{OR}=1,66)$ d'une part, et entre la parasitose intestinale et l'anémie d'autre part ( $\mathrm{p}=0,021$ et $\mathrm{OR}=1,89$ ).

L'anémie était sévère chez $9,8 \%$ des enfants parasités et modérée chez $53 \%$. Il existait un lien entre les parasitoses intestinales la survenue d'anémie modérée $(\mathrm{p}=0,001$ et $\mathrm{OR}=2,6)$. 
Tableau 4. Facteurs associés aux Parasitoses intestinales

\begin{tabular}{|c|c|c|c|c|c|c|c|}
\hline $\begin{array}{c}\text { Facteurs associés } \\
\text { aux parasitoses } \\
\text { intestinales }\end{array}$ & \multicolumn{2}{|c|}{ Résultats microscopiques } & \multicolumn{2}{|c|}{ Négatif } & Total & $\begin{array}{c}\text { P } \\
\text { value }\end{array}$ & Ods[ICà 95\%] \\
\hline Tranche âge & & & & & & 0,007 & \\
\hline Moins de 1 an & 5 & 9,4 & 48 & 90,6 & 53 & & 1 \\
\hline 1-5 ans & 49 & 28,7 & 122 & 71,3 & 171 & & $3,9[1,4-11,7]$ \\
\hline 5-10 ans & 28 & 29,8 & 66 & 70,2 & 94 & & $4,1[1,4-13,0]$ \\
\hline Plus de 10 ans & 21 & 36,8 & 36 & 63,2 & 57 & & $5,6[1,8-18,9]$ \\
\hline Malnutrition & & & & & & 0,035 & \\
\hline Oui & 38 & 34,8 & 72 & 65,2 & 110 & & $1,66[1,01-2,73]$ \\
\hline Non & 60 & 22,7 & 205 & 77,3 & 265 & & \\
\hline Anémie & & & & & & 0,001 & \\
\hline Oui & 84 & 30,6 & 191 & 69,4 & 275 & & $2,6[1,4-4,8]$ \\
\hline Non & 14 & 14 & 86 & 86 & 100 & & 1 \\
\hline
\end{tabular}

\section{Discussions}

Cette étude avait pour objectif de déterminer la prévalence des parasitoses intestinales chez les enfants reçus en consultation au service de pédiatrie de l'hôpital Roi Baudouin à Guédiawaye, dans la banlieue de Dakar. Ainsi une prévalence de $27,5 \%$ a été observée. Cette prévalence globale est supérieure à la prévalence nationale $(21,9 \%)$ indiquée dans une enquête de masse réalisée par l'OMVS en 2010 (Ministère de la santé et de l'action sociale du Sénégal, 2011). Ce qui démontre l'ampleur de ces pathologies négligées chez nos populations. De plus, il a été mentionné un léger recul de l'incidence de ces parasitoses intestinales en milieu périurbain après l'introduction du déparasitage de masse en 2006 (Ministère de la santé et de l'action sociale du Sénégal, 2011). Dans des études antérieures menées dans le même site, Ndir et Diouf (Ndir, 2001 ; Diouf, 2016) avaient trouvé respectivement en 2001 et 2015 des taux de prévalence de 56,62\% et $45,5 \%$.

Ailleurs au Sénégal, Diouf et ses collaborateurs (Diouf, 2000) avaient trouvé une prévalence de $31,1 \%$ au centre ouest du pays dans la zone de Khombole en 1997, alors que Tine et ses collaborateurs (Tine, 2013) avaient rapporté des chiffres de 30,4\% au sud Est et 26,2\% au centre du pays. En Afrique, des prévalences autour de 50\% ont été rapportés par Hamitt au Tchad (Hamitt, 2008) et Cissé au Burkina (Cissé, 2011).

Les parasites isolés à travers cette étude étaient essentiellement dominés par les helminthes. En effet, les espèces les plus fréquemment isolées 
étaient Ascaris lumbricoides. Les protozoaires intestinaux n'étaient retrouvés qu'à des proportions très faibles. Quelques individus de la population de l'étude étaient parasités par deux espèces Ascaris lumbricoides et Trichocéphales.

En 1999, Wane mettait en évidence une prévalence globale des parasitoses intestinales au niveau des populations sénégalaises vivant en zone périurbaine de l'ordre de $42,3 \%$, avec un spectre majoritairement dominé par Ascaris lumbricoides (31,4\%) (Wane, 2000). Quelques années plus tard, N'Dir avait objectivé une prévalence globale de $56,6 \%$ de parasitoses intestinales en banlieue dakaroise, avec une plus grande fréquence d'Ascaris lumbricoides $(34,2 \%)$, Trichuris trichiura $(22,1 \%)$; Giardia lamblia représentait 22,5\% (Ndir, 2002). Dans une étude réalisée en 2008, Faye avait obtenu une prévalence globale de parasitose intestinale de 44,6\% majoritairement dominée par Schistosoma mansoni (15,4\%), Ascaris lumbricoides $(12,1 \%)$ (Faye, 1998).

En ce qui concerne l'état nutritionnel, la MAS et la MAM représentaient respectivement $10,5 \%$ et $18,9 \%$. Diouf avait trouvé des proportions plus élevées de MAM (19,1\%) et de MAS (12,9\%) (Diouf, 2016). Ces prévalences de la malnutrition aigüe semblent élevées par rapport aux résultats de l'EDS-C 2016 qui retrouvait un taux de prévalence de la malnutrition aiguë de l'ordre de7 \% (EDS-C 2016). Ce qui pourrait être expliqué par le fait que cette étude se soit déroulée en milieu hospitalier souvent fréquentés par des enfants malades.

Nous avons retrouvé une prévalence assez élevée des MAM et MAS dans cette étude et leur association significative avec les parasitoses intestinales. Cette association est aussi retrouvée par Diouf et Roger en zone rural au Sénégal. Les parasitoses intestinales ont un retentissement certain sur l'état nutritionnel. Ces résultats suggèrent que les politiques nationales visant à déparasiter systématiquement les enfants suivis dans centres de récupération nutritionnelle devraient être encouragées.

Parallèlement, une anémie était notée chez 275 enfants soit $69,4 \%$. L'anémie sévère et modérée représentait respectivement $4,8 \%$ et $68,6 \%$ dans notre série. L'enquête démographique et de santé continue (EDS-C) indique qu'au Sénégal deux enfants de 6-59 mois sur trois (66\%) sont atteints d'anémie : $29 \%$ sous une forme légère, $34 \%$ sous une forme modérée et $3 \%$ sont atteints d'anémie sévère (EDS-C 2016).

Le lien entre les parasitoses intestinales et la survenue d'anémie modérée ( $\mathrm{p}=0,006$ et $\mathrm{OR}=1,69)$ retrouvée dans cette étude n'a pas été mis en évidence par Roger. Ceci peut être expliqué par la différence de l'espèce parasitaire prédominant dans les études. En effet les helminthes prédominants dans cette étude peuvent entrainer une hémorragie digestive chronique et provoquer une anémie. Au Cambodge, V. Khieu a établi une conclusion allant 
dans ce sens avec une association significative $(\mathrm{p}=0,05)$ entre l'anémie et la présence d'ankylostome (Khieu, 2006).

Cette étude s'est déroulée en milieu hospitalier souvent fréquentés par des enfants malades ceci peut constituer une limite quant à l'appréciation de la prévalence. D’autres études réalisées notamment dans la communauté en milieu suburbain pourraient permettre de mieux évaluer l'impact de ces pathologies et l'efficacité des stratégies de déparasitage ; car il apparait clair que la distribution des parasitoses intestinales diffère en fonction de la zone. L'extension du déparasitage aux enfants de plus de 5 ans et l'amélioration des conditions pourraient améliorer la stratégie de chimioprophylaxie de préventive.

\section{Conclusion}

Dans la zone périurbaine des Niayes, la prévalence des parasitoses intestinales est élevée. Notre étude montre un lien important entres ces parasitoses intestinales et la malnutrition d'une part et l'anémie d'autre part. Les résultats suggèrent que l'élargissement de la chimioprophylaxie de masse aux enfants de plus de 5 ans et l'amélioration des conditions d'hygiène contribueraient à réduire significativement la prévalence de ces maladies qui pèsent sur l'état de santé des enfants.

Remerciements : Centre d'Excellence pour la Santé de la Mère et de l'Enfant (CEA- SAMEF). Personnel de pédiatrie de l'Hôpital Roi Baudouin.

\section{References:}

1. Adam, K.M.G., Paul, J. \& Zaman, V. (1971). Laboratory aids in the diagnosis of protozoan infections. Medical and Veterinary protozoology, an illustrated guide. Longman group limited, Ontario: 162-166.

2. Agence Nationale de la Statistique et de Démographie (ANSD) (2017). Enquête Démographique et de Santé Continue (EDS-Continue). Dakar, Sénégal 2017.www.ansd.sn

3. Alum, A., Rubino, JR. \& Ijaz, MK. (2010). The global war against intestinal parasites-should we use a holistic approach International journal of infectious diseases. Official publication of the International Society for Infectious Diseases $2010 ; 14$ : 732-738.

4. Cisse, M., Coulibaly, S.O. \& Guisuemide, R.T. (2011). Aspects épidémiologiques des parasitoses intestinales rapportées au Burkina Faso de 1997 à 2007. Med Trop. $2011 ; 71$ : 257-260.

5. Diouf, J.B. et al. (2016). Persistance des géohelinthes en milieu hospitalier pédiatrique dans la banlieue dakaroise au Sénégal. Revue Cames Sante 2016 : 4. 
6. Diouf, S., Diallo, A., Camara, B., Diagne, I. et al. (2000). Parasitoses intestinales de l'enfant en zone rurale sénégalaise (Khombole). Méd Afr Noire. $2000 ; 5$ : 229-232.

7. Faye, O., N'dir, O., Gaye, O., Dieng, Y., Dieng, T. et al. (1998). Parasitoses intestinales chez les vendeurs et les consommateurs d'aliments de rue. Bull. Soc. Path. Exot. 1998 ; 91 : 169-172

8. Hamitt, M.A., Tidjani, M.T. \& Bilong, C.F. (2008). Recent data on the prevalence of intestinal in N'Djamena, Chad Republic. African Journal of Environnemental Science and Technology 2008; 2; (12).

9. Henriksen, S.A. \& Pohlenz, J.F. (1981). Staining of cryptosporidia by a modified Ziehl-Neelsen technique. Acta vet. scand. 22, (3-4): 594596.

10. Khieu, V., Odermatt, P., Mel, Y. et al. (2006). Anaemia in a school of rural Cambodia: detection, prevalence, and links with intestinal worms and malnutrition Bull Soc Pathol Exot 99(2):115-8.

11. Mcnabb, S.J., Hensel, D.M., Welch, D.F., Heijbel, H., Mckee, G.L. \& Istre, G.R. (1985).Comparison of sedimentation and flotation techniques for identification of Cryptosporidium sp. oocysts in a large outbreak of human diarrhea. J. Clin. Microbiol. 22, (4): 587-589.

12. Mentresor, A.C.D., Gyorkos, T.W. \& Savioli, L. (2004). Lutte contre les helminthiases chez les enfants d'âge scolaire : guide à l'intention des responsables des programmes de lutte. OMS 2004.

13. Ministere de la santé et de 1'action sociale. (2001). Plan directeur de lutte intégrée contre les maladies tropicales négligées. Ministère de la santé et de l'action social. 2011-2015.

14. Ndir, I., Gaye, A., Sy, M., Gaye, O. \& Ndir, O. (2002). Prevalence of intestinal parasitis at the king Baudouin health center of Guédiawaye (Sénégal) Dakar Med.2002 ; 47 : 167-171.

15. Organisation Mondiale de la Santé. (2018). La schistosomiase et les géohelminthiases: prévention et lutte. Rapport d'un Comité d'experts de l'OMS. http://www.who.int/fr/news-room/factsheets/detail/soil transmitted-helminth infections

16. Tine, R.C.K., Faye, B., Ndour, C.T., Sylla, K., Sow, D., Ndiaye, M. et al. (2013). Parasitic Infections among Children under Five Years in Senegal : Prevalence and Effect on Anaemia and Nutritional Status. Isrn Parasitol. 2013

17. Wane, A.T., Gaye, O., Diop, E. \& Dieng, Y. (2000). Etude des parasitoses intestinales chez des sujets habitant dans une zone périurbaine à nappe phréatique polluée par les nitrates d'origine fécale, au Sénégal. Cahier d'études et de recherche francophones /Sante, 2000 ; 9 :351-356. 